\title{
Sciendo
}

\section{Problems of image recognition systems associated with the possibility of its use in terrorist attacks}

\author{
Vladislav Semenyuk ${ }^{1}$, Ildar Kurmashev ${ }^{2}$, Vadim Sarsenbaev ${ }^{3}$ \\ ${ }^{1}$ North Kazakbstan State University "M. Kozybaev"-email: karina2011_12@mail.ru \\ ${ }^{2}$ North Kazakbstan State University "M. Kosybaev"-email:kig_ildar@mail.ru \\ ${ }^{3}$ North Kazakibstan State University “M. Kosybaev”-email: vadimcool314@gmail.com
}

Doi: $10.2478 /$ gssfj-2021-0004

\begin{abstract}
This article discusses systems for recognizing human faces, as well as systems for identifying flying objects. The paper deals with the main security issues related to the recognition of faces and images of objects. Today, automation systems that help to recognize an object, compare it with existing databases, can help prevent terrorist attacks, unauthorized penetrations, as well as the proliferation of biological and nuclear weapons. To date, the study of issues related to the possibility of using these systems is relevant and very much in demand.
\end{abstract}

Keywords: Pattern recognition, neural networks, security, terrorism, databases, weapons proliferation

\section{Introduction}

Automatic face recognition system is a system that can recognize a person's face in a photo or video and identify it based on the available databases. Today there is a huge interest in these systems, since these systems can solve a wide range of tasks from searching for a criminal to preventing terrorist threats. DeepFake (from deep learning - "deep learning" and fake - "fake") is a method of synthesizing a human image based on artificial intelligence. It is used to combine and overlay existing images onto videos.

\section{The algorithm creation different approaches}

Currently, there are a number of approaches to the emergence of face recognition algorithms. For example, the empirical approach that has appeared since the advent of computer vision systems is based on the rules used by a person to recognize the face of an interlocutor. It consists in excellent lighting moments of the elements of the face, the brightness of other parts of the face, in turn, is basically a monochromatic brightness. The presence of eyes, mouth, nose on the resulting image will also be a factor of recognition. To study the faces of people, the area 
where the face is located is reduced, the product of special graphs and grids is performed. These methods are easily implemented, when there are a large number of foreign objects in the image, they become meaningless, because even a change in the camera's angle of view can affect the recognition quality. Another of the methods used on the empirical approach uses a system that uses invariant features, that is, this method reveals the characteristic features of the face, its placement, contrasting areas of the face, after searching for these features, the combination and search for an image match takes place. Recognition in such cases becomes possible in the presence of an indistinct background of the image and the presence of a large number of unauthorized persons.

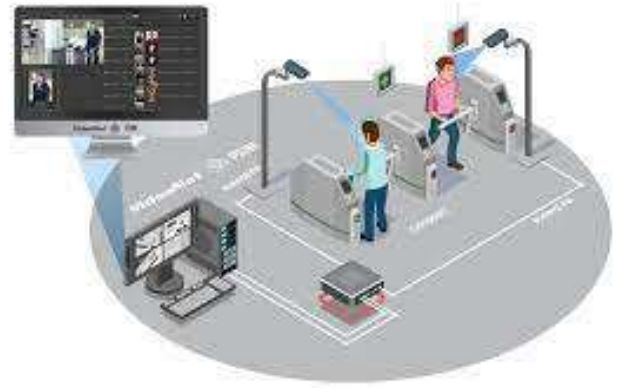

Figure 1. Possibility of using face recognition systems

Another algorithm is based on face recognition by patterns in the database, there are many face patterns and the task of the algorithm is to search for matching each segment of the image to the existing pattern, search and verification is performed regardless of the size of the images and the angle of the image. This system involves a lot of computation. Modern face examination systems are trained using test images; images of various configurations are used for training. As a rule, parts of images are described as a vector of features, using this vector, the algorithm determines whether the image is a face in the frame or not. Systems for studying faces can differ significantly in technological parameters, but they have similar principles of operation.

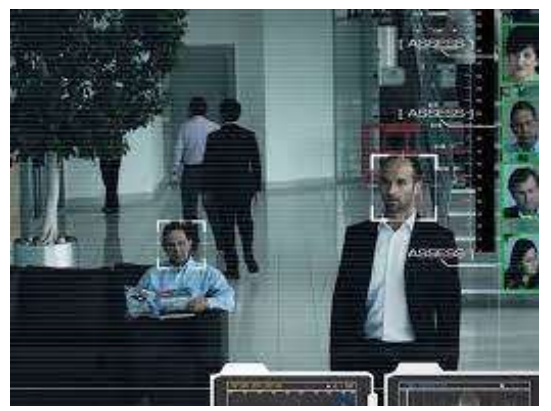

Figure 2. Search for a person by face recognition 
Technologically, systems can sometimes be very different in terms of face recognition, but they all have roughly general principles of operation(Fig3).

Step 1: Object detection. The camera searches and enters information, after which the system starts searching for a face on this image, regardless of the number of images in the image. The main secret of good face recognition in an image is the direction of a person's gaze where his gaze is directed. Modern systems allow finding a person's face in the image, if the person does not even look directly into the camera, some actions, it all depends on the circumstances.

Step 2: Explore the object. The face of the object under study is extracted from the image and analyzed. The face image is analyzed in $2 \mathrm{D}$ format because $3 \mathrm{D}$ images are less available and because, as a rule, the base is formed from 2D images. All people consist of special points, any human face contains 80 special points, thanks to the recognition program, it analyzes various distances between the eyes, nose, mouth, etc.

Step 3: Get image data. The next analysis program builds a mathematical model. After that, the analysis of the face turns into a mathematical formula, converts it into a binary code, this code is called a "face cast", each cast is individual for each person.

Step 4: Search for similar images. The following is a comparison of the existing face print with the existing base.

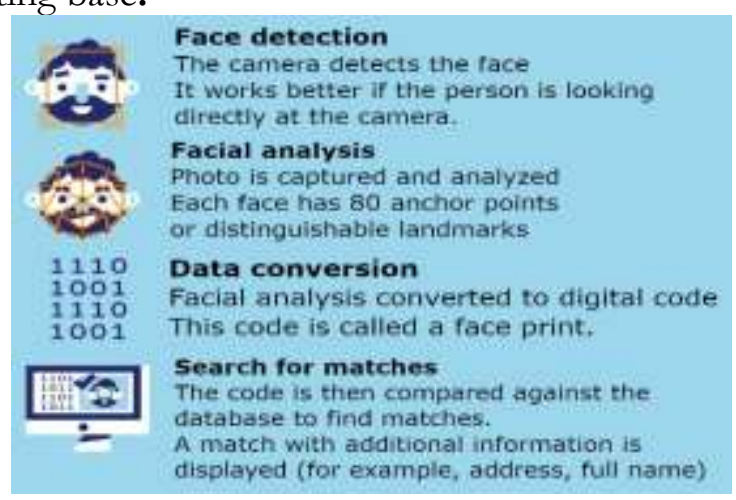

Figure 3. Face recognition system operation

Many banking systems and access control systems use face protection for data, because the best and most reliable password cannot provide protection from professional hacking. A facial cast is one of the ways to create a more secure password than a combination of symbols and letters, because a person's appearance is much more difficult to fake. These programs often request verification by confirming their appearance. Your photography also works for you. There are also systems that recognize whether a store robbery is taking place while taking a face photo. Subsequently, the system will be able to distinguish the store owner from the thief. 


\section{The process of algorithm creation}

From the point of view of intelligent information systems, the image is a collection of data about a real or abstract object (process, phenomenon), which allows one to select it from the entire set of analyzed data and group it with other objects following the requirements of the problem being solved. Requirements of a specific task avoid redundancy in the description of the image, therefore, simplifying its analysis during recognition. This is especially important in cases where, when recognizing you must process large amounts of data, which is typical for tasks of thematic interpretation of aerospace images. The characteristics of the object required to solve specific recognition tasks are called features. Often, in recognition tasks, it is necessary to distinguish several groups of objects of a certain type. In this case, it is necessary to use signs that distinguish each group from all others. Such groups in recognition are usually called classes. The task can be to select only one class (target), and everything else can be considered as the class "other". Therefore, in any case, recognition can be considered as a task classification of multiple images (Tuan T., Philpot D., 2002) From this point of view, any recognition algorithm represents is an abstract system $\mathrm{R}$, consisting of three sets:

$$
R=\langle A, S, P>
$$

where A - a list of classes to be distinguished, S - a set of features that make it possible to assign images to a particular class from the set $\mathrm{A}, \mathrm{P}$ - decision-making rules when assigning an image to a class.

The sets $\mathrm{A}$ and $\mathrm{S}$ constitute the information component of the system recognition and are closely related. Depending on how the characteristics of the classes are described, different approaches to the organization can be applied recognition process. The main such approaches are directly related to mathematical methods of describing sets:

- The principle of comparison with the standard. It is used when a class is described by one or more reference images, that is, a set of the images of one class are specified by enumerating its elements.

- Clustering principle. Matches the predicate way setting the sets - by a system of restrictions on the values of features. In this case, certain ranges of characteristic values are assigned to each class.

- The principle of the generality of properties. Corresponds to the assignment method sets by a generating procedure, which ultimately determines the properties of images - elements of a given set.

The principle of comparison with the standard is applied in cases where each class $A_{k}$, a finite set of reference images can be matched $\Omega_{k}=\left\{\omega_{m}, \mathrm{~m}=1, \ldots, M_{k}\right\}$. Therefore, the principle of comparison with the standard is differently called the enumeration principle. In this case, the recognition process consists of a simple comparison of the input images recognizing device or algorithm, with standards $\Omega_{k}$ of classes Ak, on based on the selected measure of similarity. The principle of 
comparison with the standard is one of the first approaches that have arisen in the construction of technical recognition systems when the capabilities of computing devices were very limited.

Clustering principle. If the characteristics are sets measurements (parameters) without any explicitly specified interconnections, then the image can be represented as an $\mathrm{n}$-dimensional vector in attribute space $\mathrm{X}$ :

$$
\vec{x}=\left\{\begin{array}{l}
x_{1} \\
x_{n}
\end{array}\right\}
$$

Each class $\mathrm{Ak}$ is associated with some set of vectors in space $\mathrm{X}$. As a result, the feature space is divided into areas corresponding to the classes $\mathrm{Ak}$, which are called clusters or taxa. Any number can correspond to one class region of space X. These regions may overlap, but the methodology decision-making must ultimately provide an unambiguous assignment of a point in space to a certain class.

The principle of generality of properties makes essential use of the connections between elements of the image. As a rule, it is used in cases where the set of images of each class is too large to obtain a reliable description of a finite number of standards, but it is possible to identify a sufficient number of distinctive features of classes for final samples of images. Revealed properties are coded based on the appropriate models and are stored in memory in the form of some structures, functions, or relationships. In the process of recognition, the image is analyzed according to the scheme, allowing to identify of the necessary properties of the image; then they are compared with the properties of the classes Ak. A generalizing property can be the algorithm itself for generating images; in this case, the image classes are given by algorithms for generating structures of a certain type.

Before starting to build a recognition system, it is necessary to analyze all available information about objects research and solve the following issues.

1. What are the general characteristics and properties of objects research and how do they differ?

2. If the required characteristics can be obtained as a result measurement, what is the accuracy of these measurements?

3. Is there a suitable model (s) to formally describe and analysis of these characteristics?

Based on the research carried out, the type and structure are determined by recognition systems. By the physical nature of the characteristics-signs of the images of the system, recognitions are classified into simple and complex. An example of simple recognition systems in the tasks of processing remote sensing data is a classification of pixels multispectral scanners or digitized multispectral images. In this case, the signs are spectral reflective properties of objects on the earth's surface. How only other types of data are involved in the classification process, the system becomes complicated. Complex recognition systems can be single-level and multilevel. In single-level systems, recognition is carried out based on one dictionary of features by one algorithm recognition. In multilevel systems, recognition results obtained at one stage are used as input data for the next. Most of the technologies for the thematic processing of remote sensing data when solving applied problems 
are implemented by complex multi-level recognition systems. Splitting the scheme for solving the problem into levels is called decomposition. Method of decomposition of the recognition system usually associated with the moments of inclusion in the recognition process data analyst, that is, with an interactive mode processing.

A common important characteristic of digital images of all of the listed types, affecting the magnitude of the final errors, is the size of a resolution unit (pixel) in the earth system. Following the physical foundations of remote sensing, the brightness (or color) of each image pixel characterizes the spectral reflective properties of the corresponding area of the earth's surface. Depending on the resolutions, within one pixel can be integrated varied landscape elements with different spectral reflective properties. Therefore, when recognizing only by luminance features, even if the accuracy of selecting the boundaries of objects in the image coordinate system is high, interpretation errors are inevitable individual pixels and even small objects. To reduce errors it is better to set an excessive number of classes, and then group them by taking into account structural features in the image coordinate system, as well as using cartographic and reference data.

\section{Computer image perception}

The most important measurements for face identification systems are the study of the distances between different parts of the face, such as the height and shape of the cheekbones, the length of the nose, etc. As soon as you turn your head to the side and change the angle, these parameters will change, and the program will not be able to determine that the same person is in front of it. If you need to remain incognito, you can wear glasses to hide your eyes and cheekbones, or pull a scarf over your mouth and chin - this way you can remain anonymous. This is how facial recognition systems work in two-dimensional space (Wasserman, 1992).

Humans and computers perceive images and videos differently. When you look at a photo, you instantly recognize your best friend - even if the image is not of the best quality. For a computer, such a snapshot is data from which information about the contours of objects and their color can be extracted. Although it lacks senses, it can be taught to recognize certain places and objects, such as beaches or cars. Most neural networks are composed of formal neurons (Fig. 4).

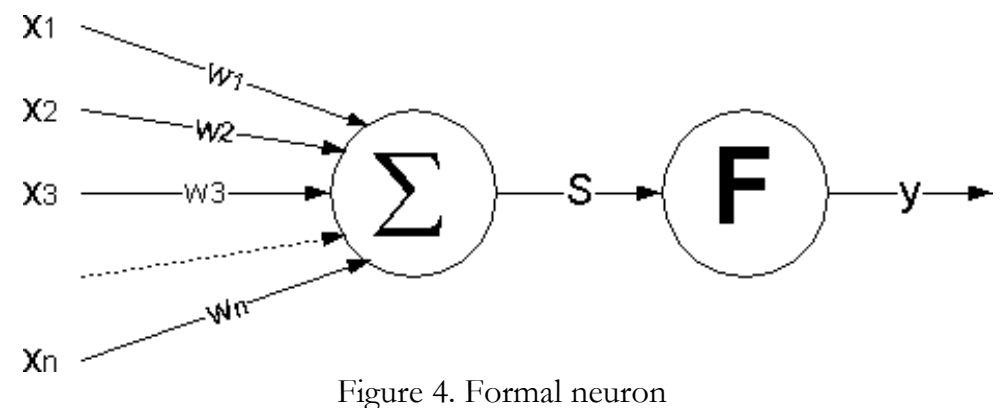


$x_{1} . . x_{n}$ values arriving at the inputs (synapses) of a neuron, w1..wn - synapse weights (can be either inhibitory or amplifying), S - the weighted sum of input

signals

$$
S=\sum_{i=1} w_{i} x_{i}-T=(w, x)-T=|W| *|X| * \cos \alpha-T,
$$

where $\mathrm{T}$ is the threshold of the neuron (in many models they do without it), $\mathrm{F}$ is the neuron activation function that converts the weighted sum into the output signal $y$ $=F(S)$. The weighted sum of the input signals can be interpreted as the projection of the input vector onto the vector of weights, where - $\cos \alpha$ the angle between these vectors.

The architecture of a multilayer neural network (Multilayer Perceptron, MLP) consists of sequentially connected layers, where the neuron of each layer is connected by its inputs to all neurons of the previous layer, and the outputs to the next (Fig. 5). Activation functions for such neurons there are varieties of linear, threshold, and sigmoid functions.

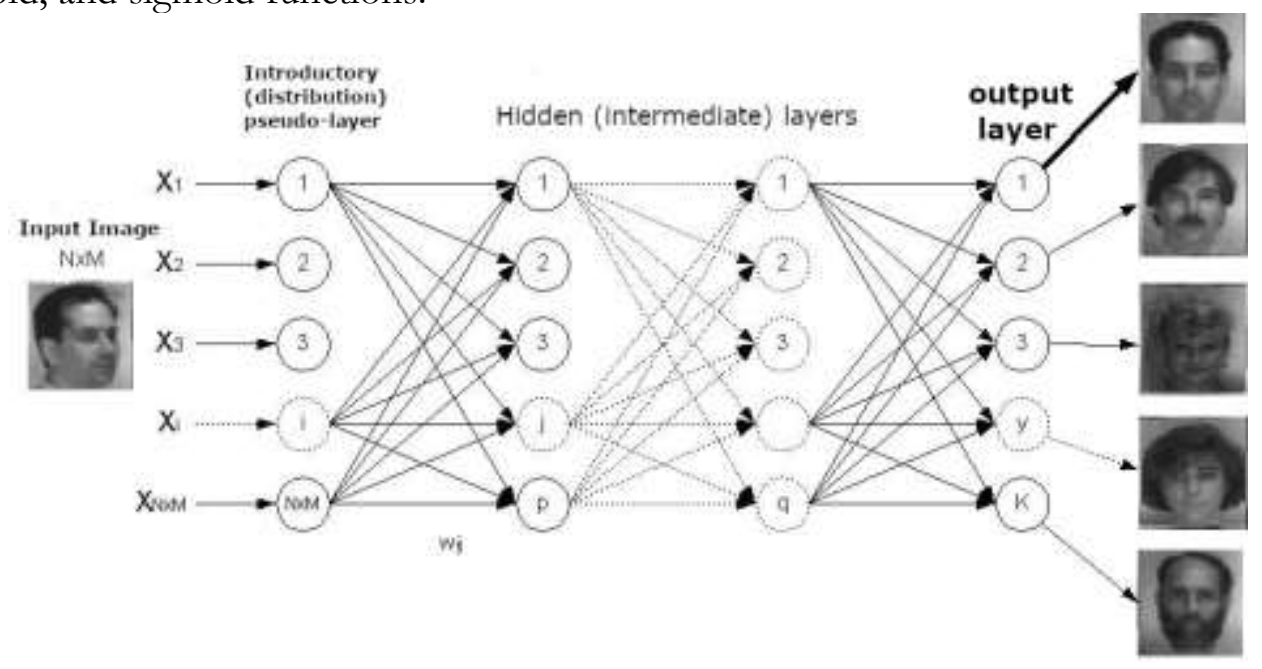

Figure 5. The architecture of a multilayer neural network and its application for image recognition. The neuron with the maximum activity (here the first) indicates belonging to the recognized class

The actual task of human recognition from the face image is access control (restriction). The use of neural networks is promising for this task. Let's formulate the requirements for such a system:

- functioning in real-time;

- flexibility of settings, simplicity, and versatility of use;

- resistance to interclass variations of the face image (os-

broadcasting, angle);

- resistance to intraclass changes in the face image 
(emotions, glasses, beards, hair, etc.);

- resistance to errors of the first and second kind and the possibility of varying the compromise between them;

- if possible, the simplicity and versatility of the developed image recognition algorithm

All these systems can also be used for recognizing flying objects, so we distinguish that, as a rule, a flying object is at a greater distance than a person and moves at a much higher speed.

It is worth noting the fact that today there are a fairly large number of systems that allow bypassing the protection of various objects, at the same time, terrorism poses a real threat, which is becoming more and more radical. Terrorists and other elements can use these systems to search for important, in their opinion, objects, and based on these systems, it is possible to create a double of a person, using his images, a person who can enter protected objects to commit a terrorist attack or crime.

\section{Conclusion}

Modern video surveillance systems often cover large areas with a large number of video cameras, which operators cannot keep track of. Therefore, computer vision algorithms are used to detect, track and identify objects. However, in modern video surveillance systems, it is not enough just to recognize graphic images - it is required to detect dangerous situations occurring with these images. You can imagine an object as a graphic image, an event - an instant action by an object or without an object, a situation - a sequence of events, that is, situation recognition - the upper level of recognition algorithms that operate with the results of graphic image recognition. In the simplest case, situations can be divided into two types: normal and dangerous. When a dangerous situation arises, it is necessary to notify the interested parties about it. A distributed video surveillance system is characterized by a large number of cameras and a significant control area, in which objects can move from the surveillance area of one video camera to another. In this regard, there is a need to develop new algorithms for recognizing hazardous situations that allow taking into account data from multiple cameras.

Modern neural networks allow searching and comparing objects; both a person and a flying object can act as an object here. The development of image recognition systems based on neural networks will not only improve access control in various countries but also find dangerous criminals on the international wanted list. Image recognition systems will allow better disclosure of a crime when these systems are used in an access control system. 


\section{References}

Kruse F.A., Lefkoff F.N., Boardman J.B., Heidebrecht K.B., Shapiro A.T., Barloon P.J., Coetz A.F.H. 1993, The spectral Image Processing Systems (SIPS) - Interactive Visualization and Analysis of Imaging spectrometer Data: Remote Sesing and Environment, V. 44, p. 145-163. 23.Richards J. Remote Sensing. Digital Image Analysis. An Introduction. Second Edition. Berlin: Springer-Verlag,1993.

Tuan Tsai and William D. Philpot. A Derivative-Aided Hiperspecrtal Image Analysis System for Land-Cover Classification. - IEEE Transaction of Geoscience \& Remote Sensing, 2002, V. 40, № 2, p.416-425.

Wasserman F., Neurocomputing: Theory and practice.- M: MEPhI, 1992. - 184 c. (http://www.neuropower.de/rus).

\section{Web references}

https://rostec.ru/news/kak-eto-rabotaet-algoritm-raspoznavaniya-lits/ 